\title{
Map symbol design: Presenting abstract topics with advanced symbology
}

\author{
Alena Vondráková* \\ Department of Geoinformatics, Faculty of Science, Palacký University Olomouc, 17. Listopadu 50, 77146 Olomouc, Czechia, Alena \\ Vondráková - alena.vondrakova@gmail.com \\ * Corresponding author
}

Keywords: map symbol design, cartographic semiology, map symbology, dialectology, visual impairments

\begin{abstract}
:
Map symbols design can be one of the most complex parts of creating a thematic map. This happens when you do not use the basic or pre-defined map symbols, but new symbols are created. And mainly when those symbols include more partial information about presented phenomena and at the same time correspond and respect principles of cartographic semiology.
\end{abstract}

The principles of cartographic semiology are important since they have a large impact on user perception and cognition. Simply, it affects how map symbols work. Therefore, cartographers work very hard creating certain symbols trying do make the process of information transfer from the map to the user most effective.

The basic principle is, that map symbols are easy to understand by the users when they make a connection between the graphic design on the map and the particular feature of the real world. And it is relatively easy to design symbols for phenomena that we see and that have clear characteristics, such as a river (blue line), a water area (blue area), a parking lot (grey area) or a museum (building symbol). It is much harder to design a meaningful map symbology for something abstract what we do not see. And this is exactly what this paper deals with. The map symbols design is presented on the example of case studies: the creation of a dialectological language atlas and the creation of tactile maps.

The research focusing design approaches to the effective creation of map symbols, that works. There are some principles to follow to make them more intuitive to the users. And there are few principles, that have to be followed because it is a cartographic rule. The primary way in which symbols are changed is through their size, shape, texture, and pattern. However, detailed semiology deals with various forms of prefixes, subfixes, the internal structure of graphic elements, and other properties that can be summarized as advanced geovisualization.

The presented outputs are part of the implementation of two different projects. The first project is the presentation of a very abstract topic (dialectology) for ordinary users, the second project is the presentation of a specific topic to people with severe visual impairments. And it is in their comparison that the beauty and complexity of cartography can be seen.

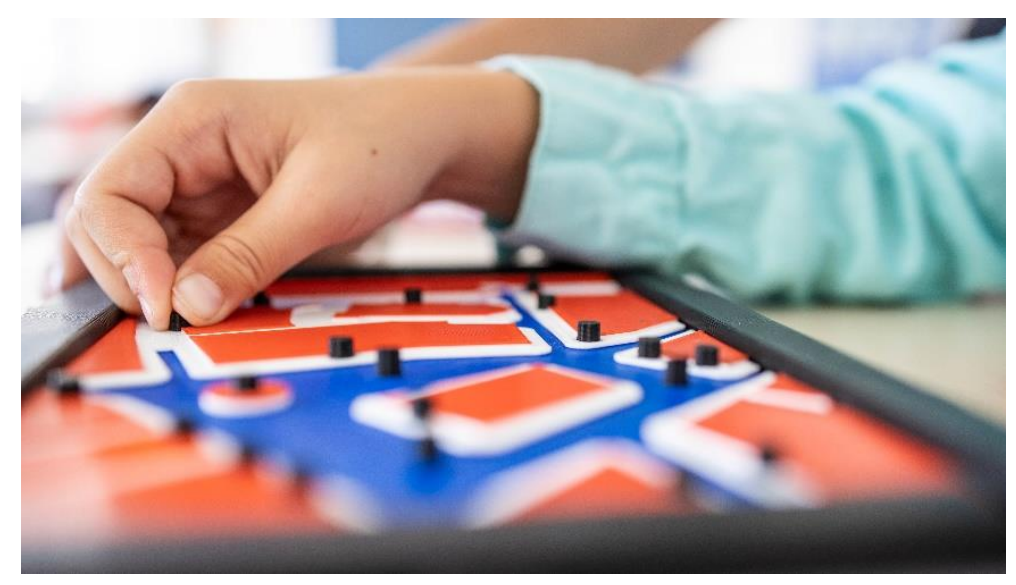

Figure 1. Testing the recognizability of individual shapes of 3D map symbols on a tactile map for people with visual impairment. 


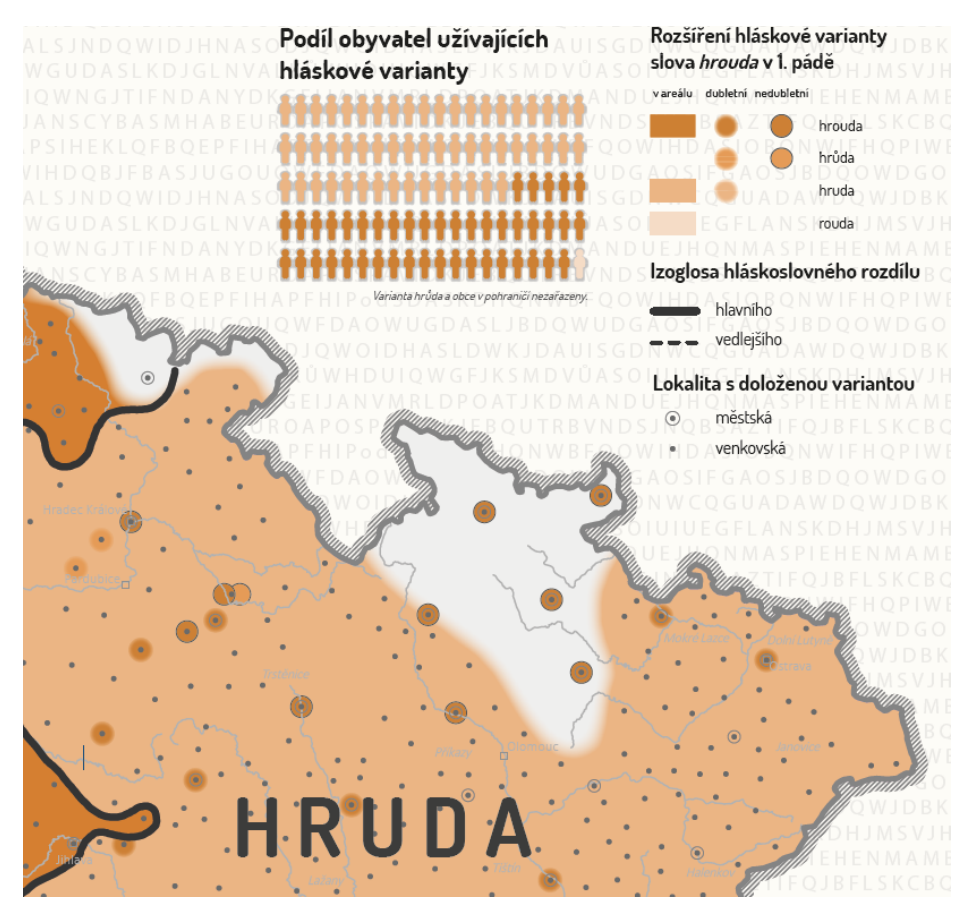

Figure 2. Example of the map legend from the dialectological map, presenting different word structure. Author: Marek Pospísili, 2020

\section{Acknowledgements}

This contribution was supported by the Technology Agency of the Czech Republic within the grant Nr. TL01000507 (research on tactile maps) and by the Ministry of Culture within the grant NAKI II Nr. DG20P02OVV029 (research on dialects). 